\title{
Microtomography and Pore-Scale Modeling of Two-Phase Fluid Distribution
}

\author{
Dmitriy Silin · Liviu Tomutsa • Sally M. Benson • \\ Tad W. Patzek
}

Received: 28 September 2009 / Accepted: 22 July 2010 / Published online: 11 August 2010

(C) The Author(s) 2010. This article is published with open access at Springerlink.com

\begin{abstract}
Synchrotron-based X-ray microtomography (micro CT) at the Advanced Light Source (ALS) line 8.3.2 at the Lawrence Berkeley National Laboratory produces threedimensional micron-scale-resolution digital images of the pore space of the reservoir rock along with the spacial distribution of the fluids. Pore-scale visualization of carbon dioxide flooding experiments performed at a reservoir pressure demonstrates that the injected gas fills some pores and pore clusters, and entirely bypasses the others. Using 3D digital images of the pore space as input data, the method of maximal inscribed spheres (MIS) predicts two-phase fluid distribution in capillary equilibrium. Verification against the tomography images shows a good agreement between the computed fluid distribution in the pores and the experimental data. The model-predicted capillary pressure curves and tomography-based porosimetry distributions compared favorably with the mercury injection data. Thus, micro CT in combination with modeling based on the MIS is a viable approach to study the porescale mechanisms of $\mathrm{CO}_{2}$ injection into an aquifer, as well as more general multi-phase flows.
\end{abstract}

Keywords Capillary pressure $\cdot$ Microtomography $\cdot$ Pore-scale modeling · Two-phase flow

D. Silin $(\varangle) \cdot$ L. Tomutsa

Lawrence Berkeley National Laboratory, 1 Cyclotron Road, MS 90R1116, Berkeley, CA 94720, USA

e-mail: DSilin@lbl.gov

S. M. Benson

Energy Resources Engineering Department, Stanford University, 074 Green Sciences Building, 367 Panama Street, Stanford, CA 94305-22020, USA

T. W. Patzek

Department of Petroleum and Geosystems Engineering, The University of Texas at Austin, CPE 2.502, Austin, TX 78712, USA 


\section{Introduction}

Geologic sequestration of carbon dioxide may reduce the greenhouse gas emissions into the atmosphere. Both, theoretical thermodynamic analysis of electricity generation at a coal-fired power plant (Apps 2006) and statistical data from the industry (Patzek 2010), suggest that capture of $\mathrm{CO}_{2}$ without significant reduction in efficiency is difficult (Apps 2006; Patzek 2010). However, redirection of the exhaust greenhouse gases into the underground may have a positive impact on mitigation of climate change (Intergovernmental Panel on Climate Change 2005). Such a solution can be viable only if there is enough confidence that the storage reservoir has sufficient capacity and that the natural seals prevent gas leakage for centuries. Injection, residence, and migration of gas and the indigenous reservoir fluids is a complex interplay of processes that occur over a wide range of scales in time and space. Only a comprehensive study addressing the problem at all scales can ultimately answer the question whether geosequestration of carbon dioxide can be a safe long-term solution. Such a study has been undertaken in Frio pilot injection project (Daley et al 2007; Doughty et al 2008). Seismic surveys, well testing, and reservoir-scale simulations focus on the large-scale phenomena. Yet two-phase fluid flow at the macroscale is a sum of a myriad of events in the individual pores of the reservoir rock. The classical models of two-phase flow (Muskat and Meres 1936; Wyckoff and Botset 1936; Leverett 1939) provide a general framework. Pore-scale models of the rock can be very advanced and sophisticated (Fatt 1956a,b,c; Blunt and King 1991; Bryant and Blunt 1992; Bryant et al 1993; Bakke and Øren 1997; Blunt 2001; Patzek 2001; Øren and Bakke 2003). Yet these are only models and the complex pore processes are still incompletely understood.

In this study, we report on an experiment where two-phase fluid distribution in the pores of a sample of natural rock has been imaged in 3D at a micron-scale resolution. The rock used in the experiment was obtained from a core acquired in the Frio pilot project mentioned above. Thus, this work complements the other studies (Daley et al 2007; Doughty et al 2008). All X-ray micro CT imaging experiments have been conduced at the advanced light source (ALS) facility at the Lawrence Berkeley National Laboratory.

A coreflood experiment on a plug of only a few millimeters in size involves a number of technical difficulties. The smallness of the core and the weakness of the rock create a challenge in selecting the imaged plug. The density contrast between the supercritical $\mathrm{CO}_{2}$ and water is low relative to the contrast between any one of the fluids and the solid grains. This circumstance creates an additional difficulty in acquiring an image with a sufficient contrast between the pore fluids. As a consequence, the computed-tomography reconstructed image includes significant noise. It can be analyzed visually, but it is almost unsuitable for a routine thresholding algorithm. Therefore, this work required customized algorithms of extraction of the pore space by segmentation combined with simultaneous elimination of small disconnected clusters. The quality of the output is yet far from perfect: it captures only major features of the pores, but entirely misses the small pores and crevices. However, it turns out that these major features are sufficient for modeling the distribution of the non-wetting fluid with the method of maximal inscribed spheres (MIS; Silin and Patzek 2006). Even though the size of the imaged sample used in simulations is small, the computed distribution of gas and water visually resembles that in the experimental data. At the same time, the injected gas almost entirely bypassed some areas and saturated some others. We associate this observation with the pore-scale heterogeneities and, probably, sample damage. The heterogeneity of two-phase saturation is observed not only at pore scale. For instance, two-phase fluid distribution in a few-centimeters core sample also can be very non-uniform (Perrin and 
Benson 2010). Nevertheless, the overall result of MIS modeling is encouraging: the calculations capture the main physics of capillarity-dominated equilibrium fluid distribution in the pores.

$\mathrm{X}$-ray computed tomography in 3D micron-scale imaging of the pore space of natural rocks was first reported in Spanne et al (1994), Auzerais et al (1996), Coles et al (1996). Coles et al $(1998 \mathrm{a}, \mathrm{b})$ published first micro CT images of two-phase (water and oil) distribution. The 30- $\mu \mathrm{m}$ resolution images acquired at National Synchrotron Light Source of Brookhaven National Laboratories were qualitatively compared to the predictions of simulations. The Lattice-Boltzmann and pore-network simulations were performed on images of dry samples of similar rock. A number of studies used micro CT imaging for investigating the geochemical transformation of the pores by stored carbon dioxide (Noiriel et al 2004; Bernard 2005; Luquot and Gouze 2009; Noiriel et al 2009; Flukiger and Bernard 2009).

Two-phase fluid distribution imaging and Lattice-Boltzmann flow simulations for packs of glass beads and natural rocks were further reported in Turner et al (2004), Prodanovic et al (2006), Prodanovic et al (2007). X-ray micro CT imaging of two-phase fluid saturation of the pores was applied to study the impact of wettability on the fluid distribution (Kumar et al. 2008). X-ray micro CT technique was used (Seright et al 2002) in to explain why gels reduce permeability to water more than that to oil in strongly water-wet Berea sandstone and in an oil-wet porous polyethylene core. A laboratory micro CT in-situ setup enabling 3D observation of multiphase fluid distribution in porous media under continuous flow conditions is presented in Youssef et al (2009). In some of the works mentioned above, the image processing includes registration of two micro CT images acquired separately. Although advanced registration algorithms have been developed recently (Latham et al. 2008), this operation imposes challenging requirement on imaging.

A distinctive feature of this work is that a carbon dioxide flooding experiment was performed at the reservoir pressure. The MIS simulations were performed on the same image of the fluid-saturated sample. Such an approach eliminates the difficulties associated with alignment and registration. At the same time, the noise in the binary data complicated image segmentation.

A number of numerical evaluations of capillary pressure curves have been undertaken on the digital images of dry Frio and Berea samples. The low level of noise in these images made it possible to use simple thresholding for segmentation. The computed capillary pressure curves for these two different types of sandstone are clearly distinguishable. Moreover, computations closely reproduce the mercury injection data. It should be recognized, however, that mercury injection experiments were conducted not on the plugs that were imaged, but on the larger cores and the data fitting required adjustment of two parameters: water saturation associated with microporosity and the effective contact angle. The first parameter characterizes the sub-resolution features of the pore space geometry which, we assume, contain the wetting fluid. The second one accounts for the uncertainty of the very definition of the effective contact angle for a fluid on a rough solid surface (Anderson 1987).

For both the sandstones, the mercury injection capillary pressure curve produced bimodal pore-sized distributions. One mode reflects the invasion pressure threshold, and the other one reflects the true pore size distribution. The uncertainties associated with distinguishing one from the other are well documented (Chatzis and Dullien 1981). The MIS method offers an opportunity of evaluation of the pore size distribution, which is free of the entry capillary pressure effects. 
This article is organized as follows: Sect. 2 describes experimental capabilities of the ALS facility used in this study. Section 3 overviews the MIS method and formulates the assumptions used in the simulations. Section 4 focuses on the two-phase fluid distribution model and compares the experimental image with the results of simulations. Section 5 describes the computed capillary pressure curves and the MIS porosimetry. Finally, Sect. 6 summarizes the findings.

\section{Synchrotron-Based microtomography at the Advanced Light Source}

The synchrotron-based microtomography is a nondestructive imaging method with a micronscale resolution. The high photon flux of the synchrotron-generated X-rays allows for considerably shorter exposure times compared to the conventional X-ray tubes. The quasi parallel beam removes the resolution limitations due to the finite size of the focal spot in the X-ray tube. Finally, the highly monochromatic beam removes the need for beam hardening corrections. For rock microtomography, X-rays energies higher than $20 \mathrm{keV}$ are necessary which are well within the range of line 8.3.2 at the Advanced Light Source at the Lawrence Berkeley National Laboratory. The principle of the experimental set up is straightforward: A parallel X-ray beam creates a radiograph of the sample on a scintillator glass which converts the X-ray photons into optical photons. The optical image is projected through an optical magnifying system onto the charge-coupled diode (CCD) chip of a high sensitivity camera. The sample rotates in small steps around a vertical axis, which in our experiment coincides with the axis of the sample, and a radiograph (projection) of the sample is captured for each step. Hundreds (or thousands) of projections are processed to generate stacks of $2 \mathrm{D}$ attenuation distributions in planes perpendicular to the axis of rotation. For micron scale resolution, the X-ray beam quality (spatial and temporal stability and homogeneity), the mechanical stability of the rotating stage and optical system have to satisfy very stringent criteria. Because the sample image has to be contained within the $\mathrm{CCD}$, the maximum object (coreholder and sample) diameter is limited by the optical system and the size of the camera CCD chip. Also, a compromise has to be made between the desired resolution, the size and number of projections and the total exposure time allowed at the beamline, as well as the total size of the files that need to be processed.

In our experiment we used a Cooke PCO4000 camera with a $4008 \times 2672$ pixels chip, each pixel $9 \times 9 \mu \mathrm{m}$ in size. The optical system provided about $2 \times$ magnification for a resolution of $4.48 \mu \mathrm{m} /$ pixel, which corresponded to a maximum $18 \mathrm{~mm}$ wide field of view. The $5.5 \mathrm{~mm}$ diameter and $20.16 \mathrm{~mm}$ long Frio sandstone sample was epoxied within an aluminum microcoreholder $(8 \mathrm{~mm}$ OD, $6.3 \mathrm{~mm}$ ID). The sample was first saturated with $0.5 \mathrm{M}$ $\mathrm{KI}$ in distilled water. Next, the sample was flooded at a rate of $0.8 \mathrm{ml} / \mathrm{min}$ with $9 \mathrm{ml}$ of $\mathrm{CO}_{2}$ at $6.9 \mathrm{MPa}$, or about 70 pore volumes.

Next, the pressurized coreholder was placed on the microtomography apparatus rotating stage. The entire sample was imaged using an X-ray beam with $35 \mathrm{KeV}$ energy in 15 vertically stacked tiles each consisting of 600 projections. Each projection was 2100 pixels wide $(9.408 \mathrm{~mm})$ and 300 pixels high $(1.344 \mathrm{~mm})$. The total vertical length scanned of $20.16 \mathrm{~mm}$ corresponded to 4,500 horizontal images which were generated by two image reconstruction software packages: Imgrec from LLNL and Octopus (Dierick et al 2004). Out of the many images, a smaller subset of 600 slices from the central region of the coreplug was selected for analysis. 


\section{The Method of Maximal Inscribed Spheres (MIS)}

\subsection{Fundamental Assumptions}

The main assumption of the method is that the pore space is fully saturated with two immiscible fluids, say, a gas and liquid water, and the system is in thermodynamic equilibrium. We neglect gravity and dynamic effects within this study. Therefore, the shape of the fluidfluid interface is determined by a minimum of the excess free energy. As a two-dimensional surface, this interface is shaped by the capillary pressure and satisfies the Young-Laplace equation (Derjagin et al. 1987), which relates the capillary pressure and the mean curvature of the surface:

$$
p_{\mathrm{c}}=\sigma \kappa
$$

Here $p_{\mathrm{c}}$ denotes the capillary pressure, $\sigma$ is the fluid-fluid interfacial tension coefficient, and $\kappa$ is the first surface curvature. The curvature of a spherical surface of radius $R$ equals twice the reciprocal of the radius, so for a spherical surface, Eq. (1) reduces to

$$
p_{c}=2 \frac{\sigma}{R}
$$

We assume that the Young-Laplace equation describes the capillary pressure between water and supercritical $\mathrm{CO}_{2}$.

The Young-Laplace equation characterizes the fluid-fluid interface in bulk fluid. Interaction of the fluids with the solid walls is affected by the wettability of the solid: a property of the solid materials to contact preferentially one fluid relative to another. The fluid that wets the solid forms a thin layer, whose stability is determined by the interaction between the fluid-fluid interfacial forces and the disjoining pressure (Derjagin et al. 1987; Israelachvili 1992). We assume that the solid is water-wet. It means that even if the water film ruptures and the gas comes into direct contact with the solid, the contact angle at the three-phase contact line is close to zero, see Fig. 1.

Given a capillary pressure, Eq. 2 determines the radius of a gas bubble in bulk water. The gas can be present in the pores in various configurations. If the capillary pressure is sufficiently high, some pores can accommodate multiple disconnected gas bubbles. The capillary pressure can be too low, so that no stable configuration will allow the gas and water to be present in the pore simultaneously. Figure 2 illustrates the idea of this assumption in a simplistic cartoon picture. The gas occupies a portion of the pore on the left-hand side, and the water occupies entirely the pore on the right-hand side and the corners of the left pore. Radius $R$ satisfies Eq. 2. We exclude dispersed gas saturation by assuming that locally the gas occupies maximal volume at the given capillary pressure. Even though each of the dashed circles depicts a spherical gas bubble that would be at equilibrium at the same

Fig. 1 The apparent contact angle between water and the solid

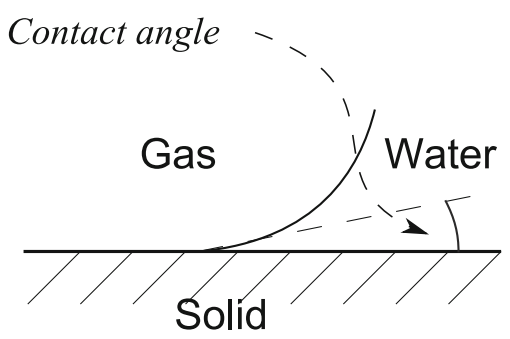




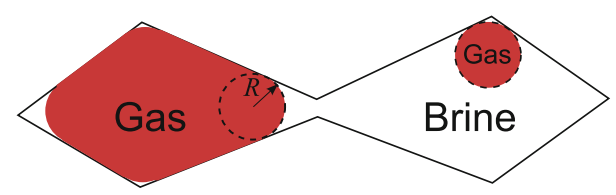

Fig. 2 A cartoon illustration of the maximal non-wetting fluid occupation: we assume that the gas saturation is like in the left-hand pore, even though isolated bubbles, like the one shown in the right-hand pore, may satisfy the capillary equilibrium as well

capillary pressure, our assumption excludes them as admissible configurations. This choice is motivated by the fact that we focus on the study of configurations imposed by fluid displacement. In drainage, we assume that if the gas overcomes the capillary entry pressure barrier, it occupies all available pore space. In imbibition, the gas phase occupancy pattern is imposed by the preceding primary drainage. Note that this maximal occupancy assumption does not determine the configuration uniquely. Indeed, in Fig. 2, the right-hand pore also can be occupied in a manner similar to the pore on the left-hand side with no violation of this assumption. At the same time, the capillary pressure determines the shape of each connected ganglion (tortuous bubble) of gas without ambiguity.

\subsection{The General Idea of the Method}

Characterization of all the connected ganglia described in the previous subsection is the main idea of the method of MIS. In Fig. 2, the gas-occupied area in the right-hand pore is the union of all circles of the radius determined by the capillary pressure through the Young-Laplace equation. Given a capillary pressure, all points inside the pores can be classified into two categories: those that can be occupied by gas, and those that cannot. The connected sets of points of the first category describe the ganglia of gas that can exist at the given capillary pressure. The points of the second category group near the corners. Whether the pores shown in Fig. 2 contain any gas, and if the gas is then present in which pore or pores, depends on the fluid displacement scenario. In primary drainage, the gas must overcome the capillary entry pressure to invade a pore. In secondary imbibition, the gas occupancy also depends on the maximum capillary pressure attained in primary drainage (Al-Futaisi and Patzek 2003). Due to the capillary entry barrier, the gas may entirely bypass some of the pores that could accommodate theoretically a bubble of gas at a lower capillary pressure.

Figure 2 only shows a two-dimensional cartoon illustration of this idea. In 3D, the situation is dramatically more complex. In $2 \mathrm{D}$, any interface of a given curvature is a circular arc. The variety of constant-curvature surfaces in $3 \mathrm{D}$ space is immensely richer. For example, a spherical surface of radius $R$ and a cylindrical surface of radius $2 R$ have the same curvature. However, the fluid-fluid interface is characterized by a minimum of the surface excess energy, or minimal area. It is known that a cylinder is not a minimal-area surface, whereas a sphere is (Pomeau and Villermaux 2006). With no geometric constraints, the minimal-area surface bounding a given volume is a sphere. Thus, in bulk fluid, the gas is in the form of a spherical bubble. However, if the bubble is confined by the solid walls of a pore, like in the left-hand pore in Fig. 2, its shape is not spherical. However, it is fair to assume that inside the pores, a spherical surface of a radius determined by Eq. (2) provides a reasonable approximation for the shape of the fluid-fluid interface. This assumption is critical for the method. It implies, that the domain that can be occupied by gas can be approximated by the union of all spherical balls of the appropriate radius fitting into the pore space. Similar to Fig. 2, the part of the 
pore volume potentially occupiable by gas consists of one or more connected ganglia. Whether the volume of a particular ganglion is indeed occupied by gas depends on the fluid displacement scenario. In drainage, only the ganglia connected to the sample inlet can be occupied by gas. In imbibition, if the preceding primary drainage has spanned practically the entire pore space, it is likely that gas will be present in all theoretically feasible ganglia. Thus, an algorithm of evaluation of a point on the drainage capillary pressure curve can be designed in two steps. First, given a capillary pressure find the union of all balls of the radius determined by Eq. 2. Second, evaluate the relative volume of the balls which are the parts of ganglia connected to the inlet. This volume gives an estimate of the saturation, which along with the capillary pressure yields a point on the desired curve. For secondary imbibition following primary drainage spanning the entire pore space, the saturation can be estimated by accounting for all ganglia, including those not necessarily connected to the sample inlet.

Two gas bubbles may share the same pore body and be separated by a water film. The stability of such films and the likelihood of coalescence is beyond the scope of this study. We assume that the total volume of water in these films is small and does not significantly affect the estimate of saturation.

In imbibition, a trapped cluster of gas may be at a pressure different from the rest of gas. In such a case, we apply a convention that the capillary pressure is determined by the pressure of gas connected to the inlet.

\subsection{An Implementation of the Method}

The above-described model can serve as a tool for simulating equilibrium two-phase fluid distribution and numerical evaluation of the capillary pressure curve. A computer tomography image of the pore space of a rock sample can be used as input data.

Pore-scale modeling of porous media employs similar approaches. The simplicity of the idealized geometry of the flow channels makes possible simulation of a wide variety of multi-phase fluid displacement scenarios (Bakke and Øren 1997; Xu et al 1999; Blunt 2001; Knackstedt et al 2001; Patzek 2001; Øren and Bakke 2003; van Dijke et al 2007). However, this simplicity is compensated by the difficulty of generating a network of pore bodies and pore throats, which would adequately represent the pore space of a particular rock sample. Even though several algorithms transforming a digital CT image of a rock sample into a network of channels have been developed (Vogel 1997; Lindquist and Venkatarangan 1999), this transformation still remains a challenge.

A digital image is a set of cubic voxels. Each voxel has an intensity recovered from the $\mathrm{X}$-ray scans by computer tomography. A segmentation algorithm classifies the voxels into solid and void. A comparison of a number of different segmentation algorithms presented in Sezgin and Sankur (2004) shows that this operation involves uncertainty. Here, we assume that the thresholding has been already done and the image consists of only void and solid voxels.

To characterize the ganglia of inscribed balls in the pore space, we evaluate the maximal radii for all pore voxels. The result is a three-dimensional table of numbers. The algorithm used in the computations works in the following way. First, for each voxel, one determines the radius of the maximal sphere inscribed in the pore space and centered at this voxel. As a result, the entire pore space is covered with such spheres. Note that each voxel can be covered by multiple spheres: the one centered at this voxel and, perhaps, other ones centered at other voxels. For each voxel, we assign the maximal radius of the spheres covering it. This operation can be optimized by going from the largest sphere to the smallest one. 
Fig. 3 Example: three discrete spheres in $2 \mathrm{D}$

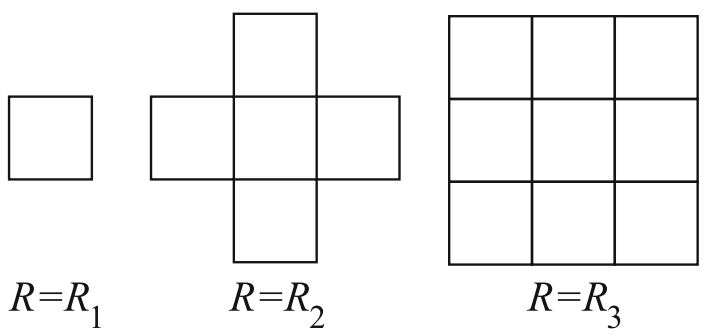

Fig. 4 An example of maximal-inscribed spheres calculations

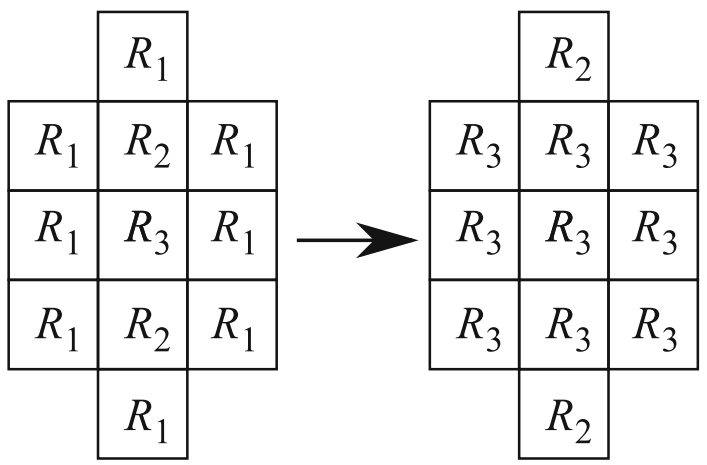

Due to the digital nature of the image, spheres must be replaced by their digital analogues. A digital sphere of radius $R$ centered at a given voxel $V_{0}$ can be defined as the set of voxels, for which the distance to the center voxel, $V_{0}$, does not exceed $R$. Figure 3 shows a cartoon example of three spheres in two dimensions. Application of the first step of the algorithm described above to a group of pixels shown in Fig. 4 results in radii distribution shown on the left-hand side picture. The discrete spheres of radius $R_{2}$ cover the top and the bottom pixels, whereas all other pixels are covered by a sphere of radius $R_{3}$ at the center. So, the second step of the algorithm yields the radii distribution shown in Fig. 4 on the right-hand side.

If the unit length is equal to the size of one voxel, the square of the radius of a discrete sphere is always an integer number equal to a sum of squares of three integers. Therefore, the radius of a discrete sphere cannot take an arbitrary real value. Equation 2 implies that the range of all possible capillary pressures computed by the method of maximal inscribes spheres is also discrete.

Computation of the part of the pore space occupied by gas follows from the calculations outlined above. Namely, the pores associated with a certain radius connected to the inlet face or faces are assumed to be occupied by invading gas. The derived relative number of occupied voxels yields an estimate of the corresponding gas saturation. The radius determines the corresponding capillary pressure through the Young-Laplace equation (2). Figure 6 shows two distributions of gas in the pore space of the sample shown in Fig. 5 computed at two water saturations. This calculation does not involve any fluid displacement scenario. At $S_{\mathrm{w}}=55 \%$, the gas phase is percolating.

Water distribution is computed indirectly, as a set of pore voxels complementary to the gasoccupied voxels. Figure 7 shows the distributions of water complementing the distributions of gas shown in Fig. 6.

The algorithm performs stably with respect to small noise in characterizing the skeleton. However, if just one voxel located in the middle of a pore has been erroneously marked as 

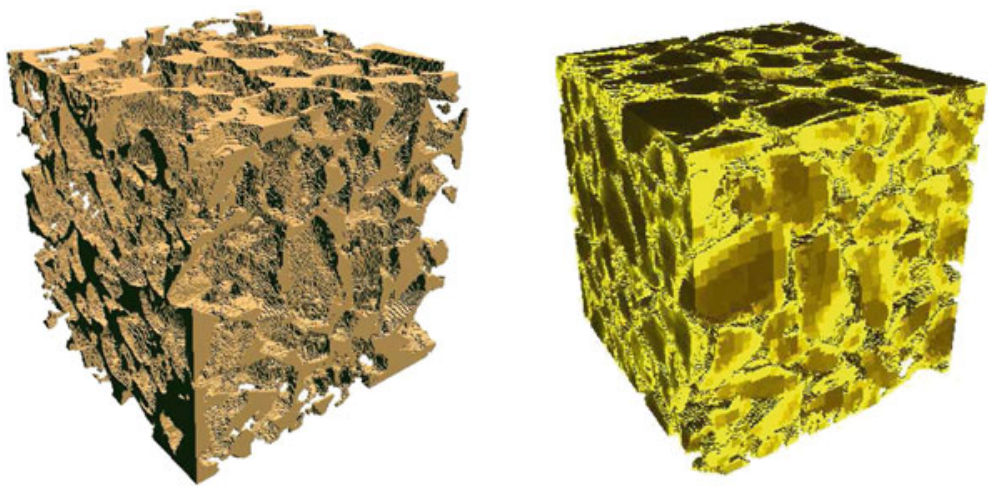

Fig. 5 An image of the pore space and the skeleton of a $0.9 \times 0.9 \times 0.9 \mathrm{~mm}^{3}$ Frio sandstone sample
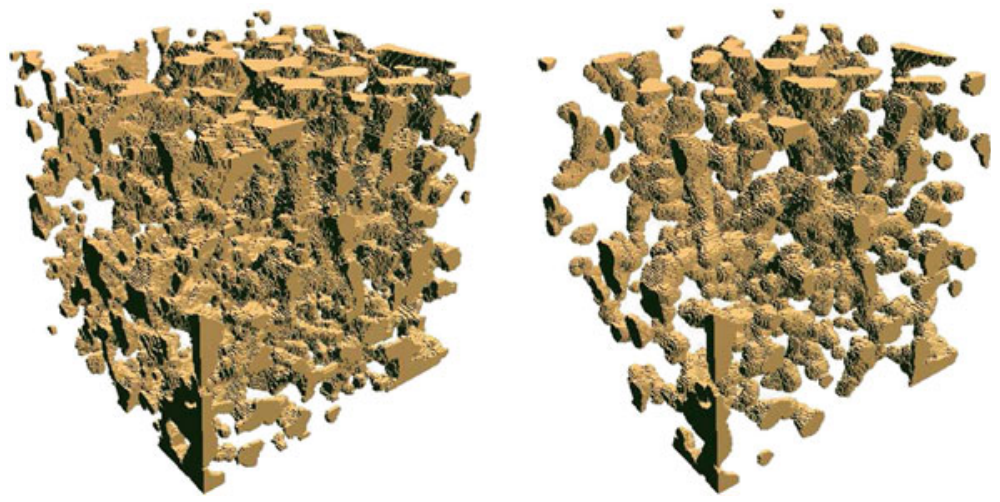

Fig. 6 Distributions of gas in the pore space shown in Fig. 5 at two water saturations: $S_{\mathrm{W}}=27 \%$ and $S_{\mathrm{W}}=55 \%$
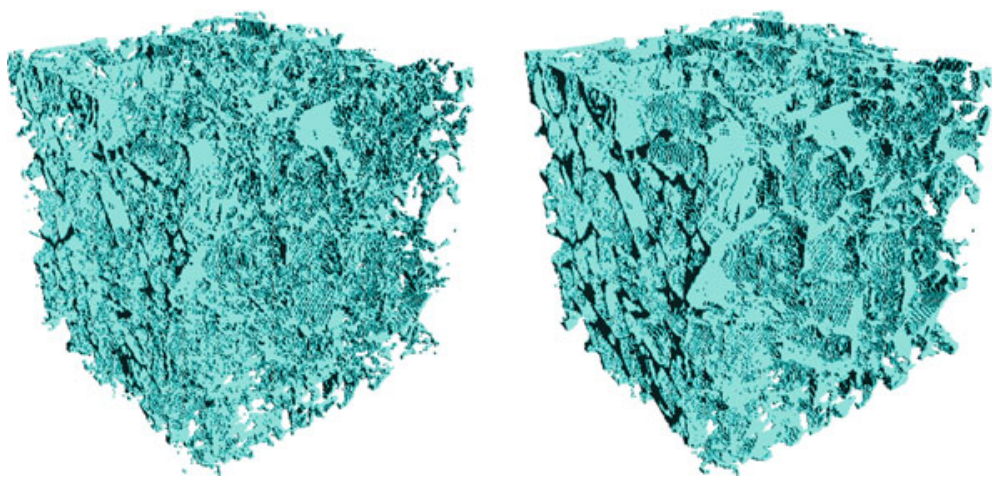

Fig. 7 Distributions of water in the pore space shown in Fig. 5 at two water saturations: $S_{\mathrm{W}}=27 \%$ and $S_{\mathrm{W}}=55 \%$ 
solid, the MIS method will produce a significant error. Therefore, an image cleanup procedure preceded all MIS-calculations reported in this study. The cleanup consists of detection of all isolated clusters of solid voxels. Disconnectedness of the solid phase in the CT image is an artifact of the reconstruction: no solid particles float in the pores. To detect the "hanging" clusters of solid material, we employed a version of the depth-first cluster search algorithm described in Silin and Patzek (2006).

\section{Pore-Scale Verification of the Model}

Visualization of the calculations described in the previous section provides useful insights into the nature of equilibrium two-phase fluid distributions in the pore space. A verification of the model requires a two-phase flow experiment, where the measurements can be performed at the pore scale. Such an experiment performed at the ALS is described in Sect. 2.

\subsection{Simulations}

The algorithm verification is as follows. First, determine the solid and the pre voxels from the reconstructed CT data. Then, use the obtained 3D image as the input data to run MIS simulations. The output of such simulations include a series of fluid distribution images at different capillary pressures. Finally, compare the computed fluid distribution with the fluid distribution in the image. Since the capillary pressure and water saturation in the image acquired at the ALS were unknown, the latter task also included evaluation of the capillary pressure, which was done by selecting the computed fluid distribution matching the CT data the best.

\subsubsection{Extraction of the Image of the Pore Space}

To obtain a 3D image of the pore space, one simply needs to identify the voxels corresponding to gas and water as pore voxels. This seemingly routine segmentation task is not so simple. The images of the water- and carbon dioxide-saturated sample have been distorted by significant noise. Figure 8 shows a two-dimensional cross-section of the sample. The darkest pixels correspond to gas, the lightest pixels show water, and the gray pixels in-between show solid. Even though the visual impression of the distribution of the fluid phases and the locations of the solid grains is reasonable, computer simulations are difficult. Each dark cluster includes a large number of gray and even white voxels. The histogram of the cross-section shown in Fig. 8 shows a unimodal distribution with no peaks associated with either phase, see Fig. 9. Smoothing the image only reduces the contrast, but does not eliminate its spotty nature.

A number of customized image-denoising routines have been developed to overcome this difficulty. The detailed fragment on the right-hand side of Fig. 8 shows that the white and gray pixels in the areas presumably occupied by gas are grouped into relatively small clusters. The same holds true for the water and solid clusters. So, such clusters can be eliminated by putting a threshold on the size. This operation also requires some initial segmentation thresholds eliminating the gas and water phases. The size of the cluster and the threshold values were selected by trial and error and visual comparison with the source image.

Figure 10 shows that the removal of small clusters does not produce a satisfactory result. Some small clusters occur near the boundaries between the solid and void. Thus, formally, such clusters are not isolated. The encircled area in the right-hand-side picture in Fig. 10 


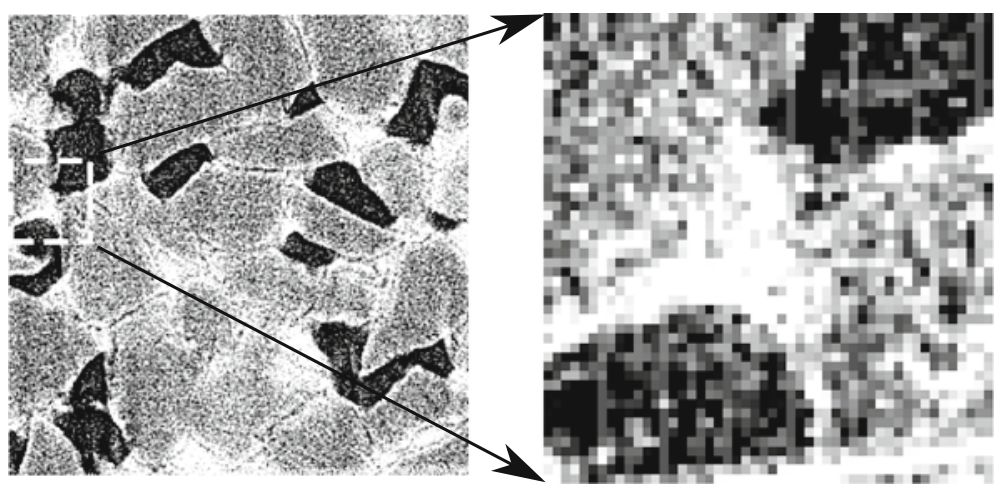

Fig. 8 A cross-section of the source image. The dark areas are gas, the white areas is water, and the gray color is solid. The zoomed detail shows the character of the noise

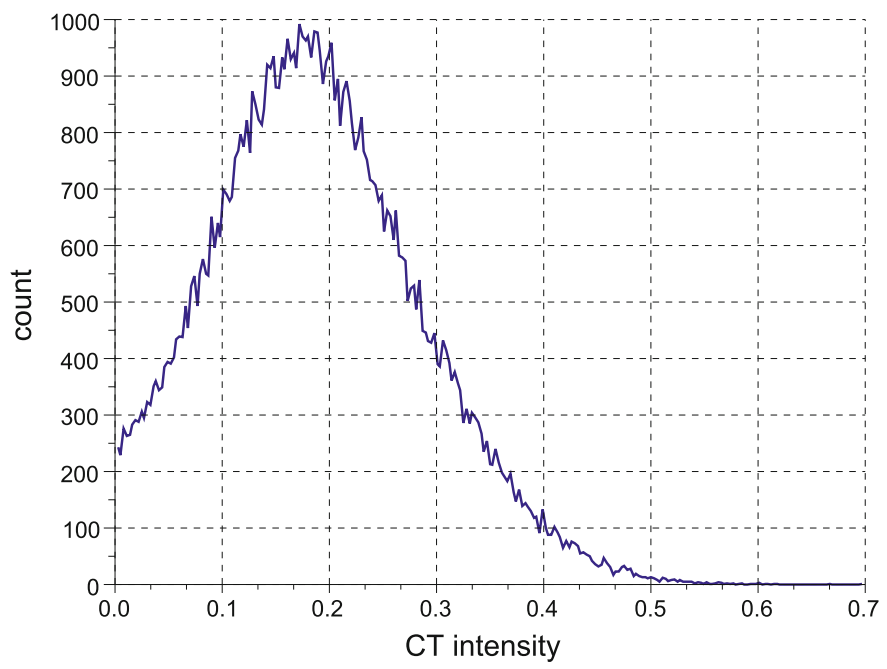

Fig. 9 The histogram of the cross-section shown in Fig. 8 exhibits no peaks or minima which would clearly indicate a threshold

examplifies such a structure. In many cases, the cluster connections are one-pixel wide. Therefore, the one-pixel connections must be detected and associated with the other phase. After this operation, the cluster search is repeated, and the small clusters that become isolated after cleaning up the 1-pixel connections are removed. This operation is iterated until no isolated clusters or 1-pixel connections are left.

The cleanup iterations described above have been applied to each individual slice of the 3D image. After stacking the slices and obtaining a 3D structure, another small-cluster removal operation has been applied, in this case the connectivity of the voxels was tested in $3 \mathrm{D}$. The threshold size of the minimal cluster has been found by trial-and-error using visual inspection for quality control. After the pre-processing, the porosity of the sample was equal to $21.7 \%$. 


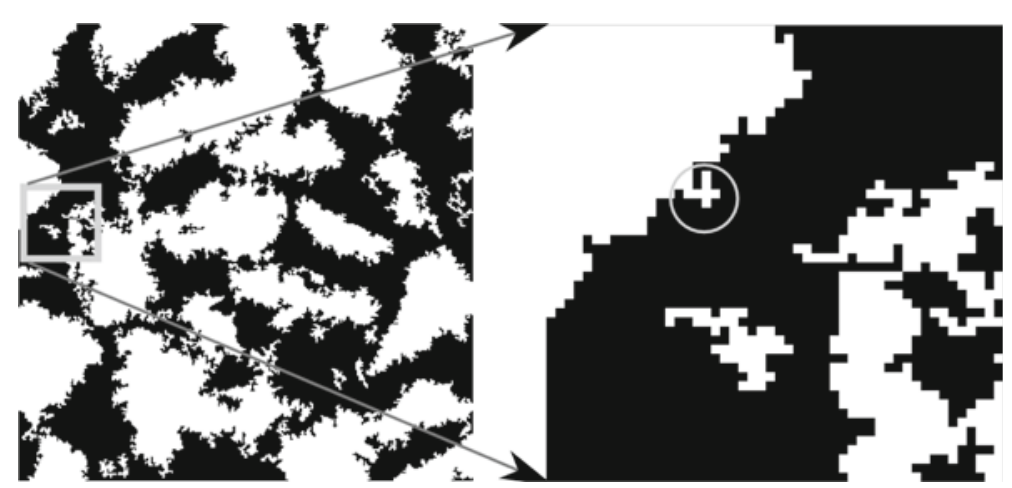

Fig. 10 A segmented image after cleaning up small clusters. White pixels denote grains and black pixels pores
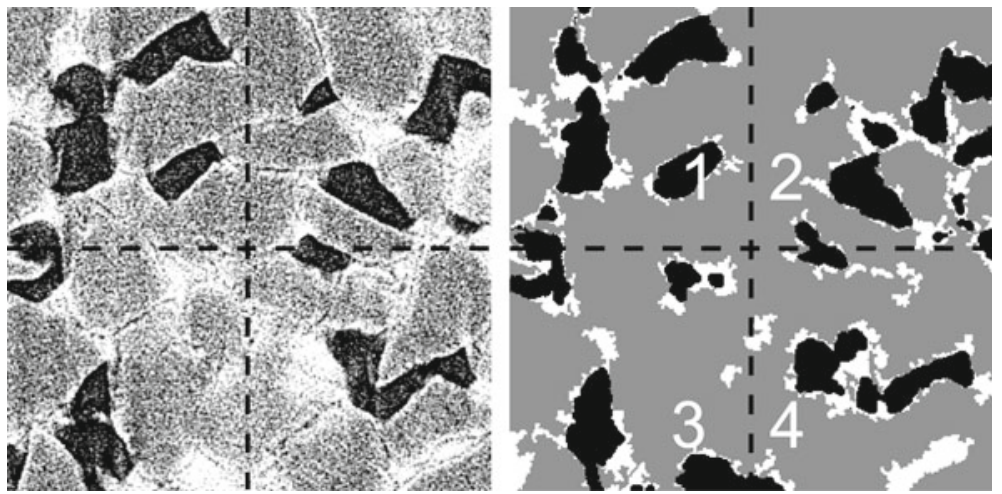

Fig. 11 Measured (left) and computed (right) fluid distributions in a 2D cross-section of the sample. The black color denotes gas, the white color denotes brine, and the gray color denotes solid skeleton

\subsection{MIS Simulations}

The two pictures in Fig. 11 shows the same cross-section of the sample. The left-hand-side picture shows the original image, and the picture on the right-hand side shows the simulation results. Apparently, some narrow gaps between the grains have disappeared. It is an artifact of image preprocessing. The simulation modeled a directional gas invasion. The best CT data matching was achieved when the invasion was orthogonal to the cross-section shown in Fig. 11, which is in agreement with the experimental settings, see Sect. 2. The computed water saturation was estimated at $71.6 \%$ at a capillary pressure near $3312 \mathrm{~Pa}$, assuming the interfacial tension of $7 \times 10^{-2} \mathrm{~N} / \mathrm{m}$. The cross-section in Fig. 11 shows $40.5 \%$ water saturation. The discrepancy between the water saturation evaluated from the entire 3D image and the estimate from a single cross-section can be explained by the fact that gas propagation has not reached the entire depth of the 3D pore geometry. Attempts to achieve a reasonable match of the source image by playing other fluid displacement scenarios have not succeeded.

Both pictures in Fig. 11 are two-dimensional cross-sections of three-dimensional configurations. This explains the variability of the gas-water interfaces curvatures. Some pores occupied by gas in the source image are gas-free in the simulations, and vice versa. 

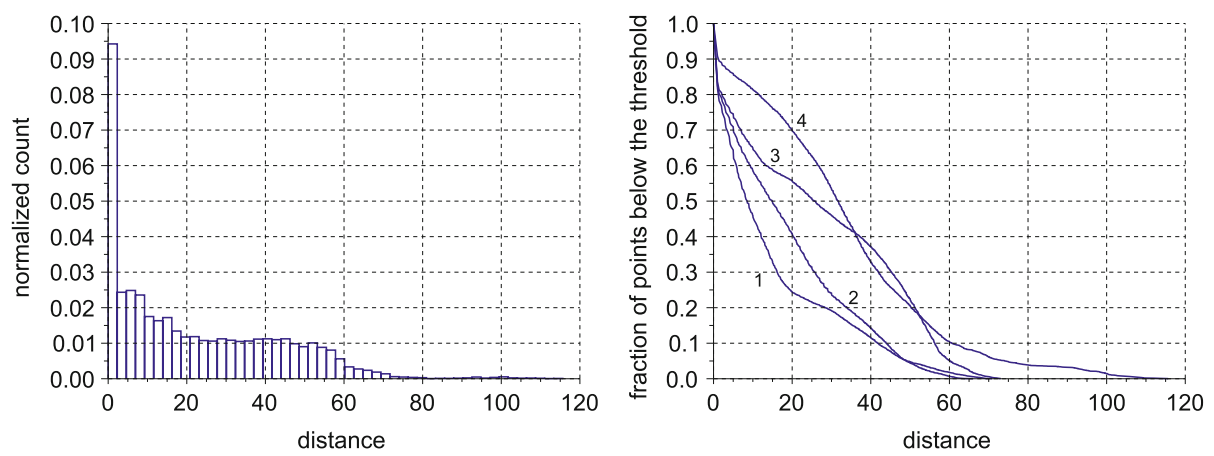

Fig. 12 A comparison of the thresholded digital data to the computed gas-occupied voxels shown in Fig. 11. The left-hand-side histogram shows the distribution for the threshold of 0.07, cf Fig. 9. The right-hand-side plot shows the portion of the points in the digital image within the threshold, which are outside the Hausdorff neighborhood of the computed gas-occupied clusters. The abscissa shows the radius of the neighborhood in voxel units

Most likely, the reasons for such a discrepancies are in the small number of the stacked slices and the limitations imposed by finite-resolution imaging and the uncertainties of segmentation.

The Hausdorff distance can serve as a measure of the difference between two images (Huttenlocher et al. 1993) and, therefore, quantify the goodness of evaluation of fluid distribution in the pores. The Hausdorff distance between two sets, $S_{1}$ and $S_{2}$, can be defined as the minimal radius such that the union of all spheres centered in the set $S_{1}$ also covers the set $S_{2}$ and, vice versa, the union of all spheres centered in the set $S_{2}$ also covers the set $S_{1}$, see, for example, Silin (1997). The character of the noise, Fig. 8, makes a formal application of the Hausdorff's distance useless since voxels fitting practically any threshold are distributed over the entire image. Instead, we analyze the distribution of the number of voxels in the digital data, which are outside a Hausdorff neighborhood of the computed cluster of gasoccupied voxels. The left-hand-side histogram in Fig. 12 shows such a distribution based on the threshold of 0.07 , Fig. 9. The radius is measured in voxels. The horizontal axes show the radius, and the count is the number of covered voxels. The largest peak is at zero, meaning that the computed gas-occupies voxels mostly cover the gas voxels in the digital image. The local peaks at $r>0$ indicate the locations where the digital image does show gas in the pores, whereas the computed fluid distribution does not. The long tail is the consequence of the noise in the digital image.

The right-hand-side plot shows the relative number of voxels of the digital image, which are within the threshold constraint, but are outside a Hausdorff neighborhood of the computed gas clusters. The distance is measured in voxel units. The four curves have been evaluated for the fours quadrants of the image in Fig. 11. The computations in quadrant 1 are most successful: $75 \%$ of the image points are within a 20-voxels Hausdorff neighborhood of the computed cluster. The least successful is quadrant 4 , where the shape of the gas cluster in the center replicates the digital image only very approximately. In all cases, $80 \%$ of voxels occurred within a 50 -voxel $(0.22 \mathrm{~mm})$ Hausdorff neighborhood of the computed cluster.

\subsection{Discussion}

The computation described in this section included two components: image preprocessing and MIS simulation. Each operation may introduce artifacts and uncertainties in the 
analysis. In the cleanup phase, the reliance on visual inspection as quality control tool makes the analysis subjective. Both isolated cluster search and the removal of 1-pixel connections may have created a number of false positives by filling up small crevices which are physically present in the sample. So, the segmented image captures only major geometric features of the pore space. In addition, only an image of a relatively thin layer of the entire sample is available for modeling. Still, such an approach to segmentation performed reasonably well in computation of the distribution of gas. Thus, MIS-based calculations of the equilibrium nonwetting phase distribution are robust with respect to the uncertainties of thresholding and segmentation. However, a single isolated solid voxel inside a pore would be detrimental to the method, and computation of a capillary equilibrium fluid distribution would be hardly possible if the data discussed in this section were not preprocessed.

Matching the data by simulations in Fig. 11 is imperfect. However, the MIS simulations have successfully captured some major features of the two-phase fluid distribution in this experimental verification of the pore-scale model. At any given capillary pressure, the MIS simulations assume that the invading fluid can enter any sufficiently large opening at the bounding faces. However, the studied volume is only a part of the entire core sample. Therefore, even for some large openings, the invading $\mathrm{CO}_{2}$ does not necessary percolate from the very inlet of the core. This circumstance is the most likely explanation why the simulations do not explain why the gas almost entirely bypassed some regions of the sample.

\section{Computed Capillary Pressure Curve and Porosimetry}

The MIS algorithm can be applied to compute a capillary pressure curve, whose shape reflects pore sizes and the pore space geometry of the sample.

Although the capillary pressure in a porous medium saturated by two immiscible fluids in equilibrium is determined by the whole history of fluid flow and distribution, it is common to characterize it as a function of the saturation, $S$ (Leverett 1941). Leverett's $\mathcal{J}=\mathcal{J}(S)$ function is a dimensionless representation of the capillary pressure of the rock (Leverett et al 1942):

$$
p_{c}(S)=\sigma \sqrt{\frac{\phi}{k}} \mathcal{J}(S)
$$

where $\sigma$ is the surface tension coefficient at the water-gas interface, and $k$ and $\phi$ are the absolute permeability and porosity of the sample.

According to the Young-Laplace equation, Equation 2, the capillary pressure can be interpreted as the radius of a spherical bubble of the non-wetting fluid multiplied by a scaling factor, which must reflect the specifics of the pore space geometry of the sample. The MIS computations produce an alternative dimensionless capillary pressure curve. Such a curve can be used as a statistical-geometric characteristic of the pore space (Silin and Patzek 2006). However, Tomutsa et al (2007) have demonstrated that after appropriate dimensional scaling, such a curve can accurately predict an experimental capillary pressure curve. The input data in the cited work were at a 20-nanometer resolution, focused ion-beam image of the North Sea chalk. Here we compare the capillary pressure curves computed from CT images of Frio and Berea sandstones with mercury injection data. Even though the sizes of samples used to acquire images at the ALS and the size of the core used in the mercury injection experiments were dramatically different, the computed and rescaled drainage capillary pressure curves predict the experimental curves amazingly well. 


\subsection{Simulations}

To reproduce a mercury injection experiment, drainage capillary pressure curves must be calculated. It means that after assigning the maximal radii in the MIS algorithm, only the voxels connected to the inlet faces are accounted for in evaluation of the saturation. Given a radius of inscribed sphere, the number of voxels with equal or greater assigned radii connected to the boundaries of the sample divided by the total number of pore voxels gives an estimate of the saturation.

There is a number of ways to define voxel connectivity. Two voxels can be called connected if they have a common face (6-connectivity), a common edge (18-connectivity), or a common vertex (26-connectivity). To cleanup the image and to remove disconnected clusters of solid voxels in pores and pore voxels in the solid, we use 6-connectivity. This strict connectivity requirement is justified by the fact that the voxels in discrete spheres are 6-connected, and even a single voxel connected to the solid phase only through an edge or a vertex creates a significant perturbation in the MIS calculations. To simulate drainage, the 18-connectivity has been used. The reason for this choice was that otherwise certain paths that are narrow due to the voxel size resolution may become blocked.

\subsection{Results}

To reduce the uncertainty associated with the smallness of the sample and to reduce the computer memory requirements, the simulations have been performed on a number of subimages. Similar computation results obtained on different samples indicate the sufficiency of the sample size.

The simulations assume zero contact angle. However, the mercury injection experiment reported a contact angle of $140^{\circ}$. The contact angle is usually measured on an ideal smooth solid surface. The roughness of natural rocks affects the contact configuration resulting in a significant uncertainty (Anderson 1987; Bico et al 2001). The dimensional scaling factor was chosen to reasonably match the capillary entry pressure.

Figures 13 and 14 show the results of computations and the mercury injection data for the Frio and Berea samples. The left-hand-side plots show the results with no calibration of the model. A number of mercury injection experiments for Frio sandstone reported in the classical paper (Purcell 1949) show, in general, higher capillary pressures for the same saturations. At low water saturations, the computed curve deviates from the experimental one more significantly than at higher saturations. Apparently, the image cannot resolve the small pores and crevices in the solid skeleton. These are the locations most likely occupied by the wetting fluid, water in our case. Therefore, the MIS calculations may miss some wetting fluid in the estimation of the fluid distribution. This deficiency does not manifest itself in the pore-scale verification in the previous section, since both the data and computations rely on the same voxel resolution. If a mercury injection experiment reaches a high pressure, the capillary pressure curve accounts for the small and tiny pores as well. This problem can be partially treated by rescaling the computed capillary pressure plots assuming some "hidden" water saturation. The right-hand-side plots in Figs. 13 and 14 show the corrected plots by assuming $22 \%$ beyond-resolution water saturation for the Frio and Berea samples. That is, the saturation in the right-hand-side plots is evaluated by the formula: $S=S_{0}+\left(1-S_{0}\right) S_{\mathrm{MIS}}$, where $S_{0}=0.22$ and $S_{\mathrm{MIS}}$ is the saturation evaluated by the method of MIS. 

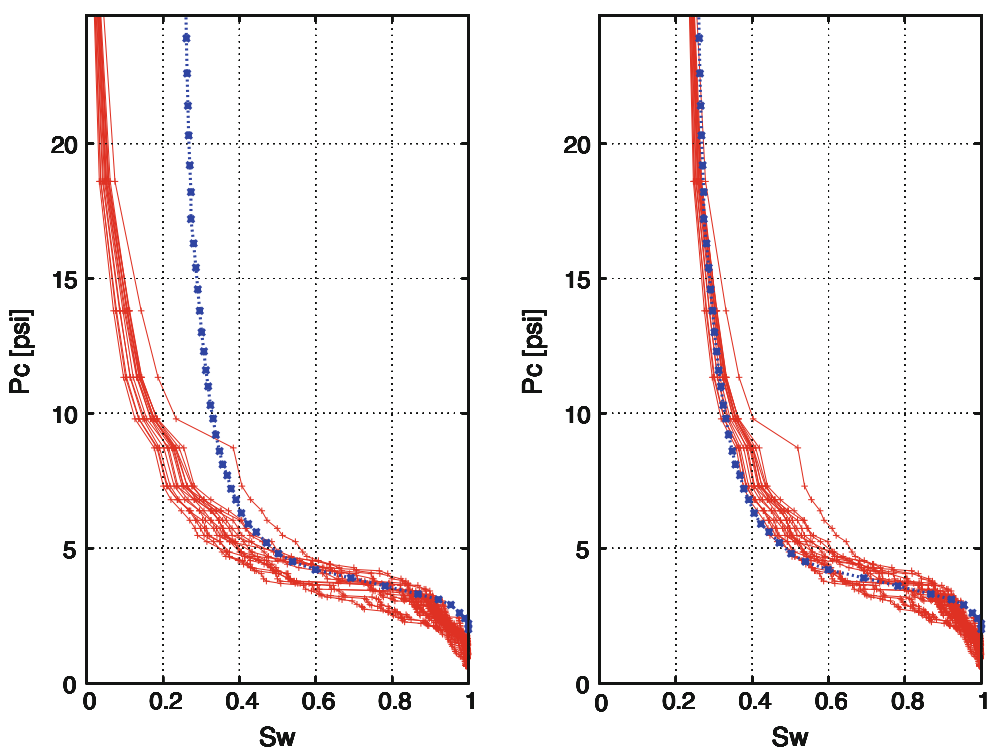

Fig. 13 The computed (solid line) and measured (dotted) drainage capillary pressure curves for a Frio sandstone sample. The markers on the curves mark the data points. The left-hand-side plot shows computed curves with no adjustments of parameters, whereas the right-hand-side plot shows computed curves rescaled for assumed $22 \%$ water saturation not captured because of the resolution limitations
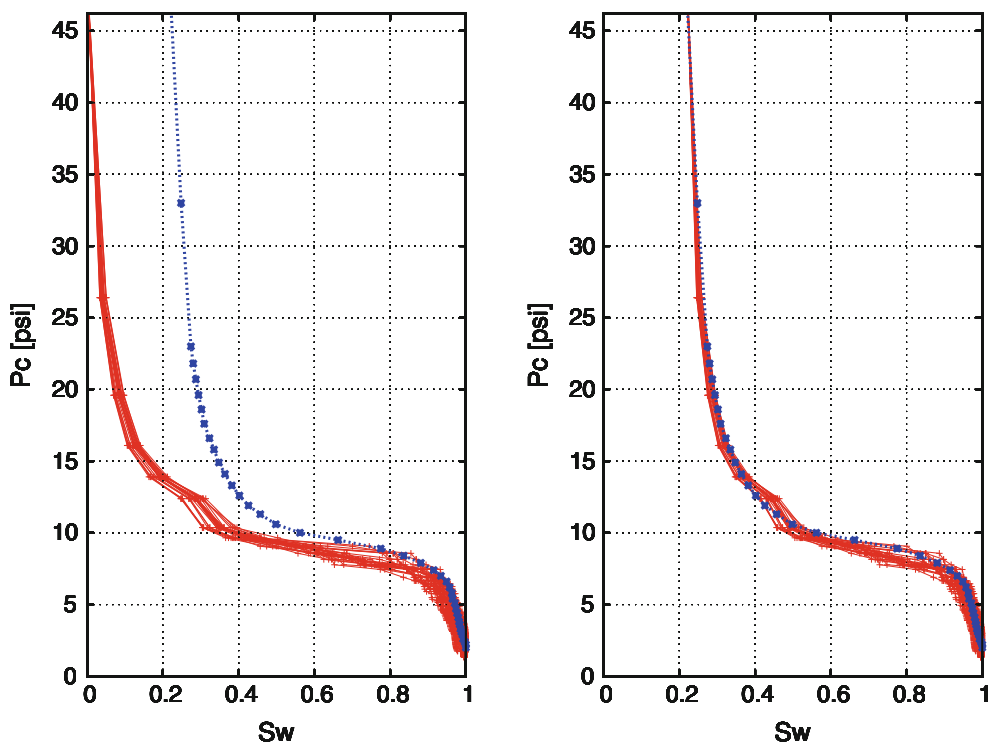

Fig. 14 The computed (solid line) and measured (dotted) drainage capillary pressure curves for a Berea sandstone sample. The markers on the curves mark the data points. The left-hand-side plot shows computed curves with no adjustments of parameters, whereas the right-hand-side plot shows computed curves rescaled with the assumed $22 \%$ water saturation not captured because of the resolution limitations 

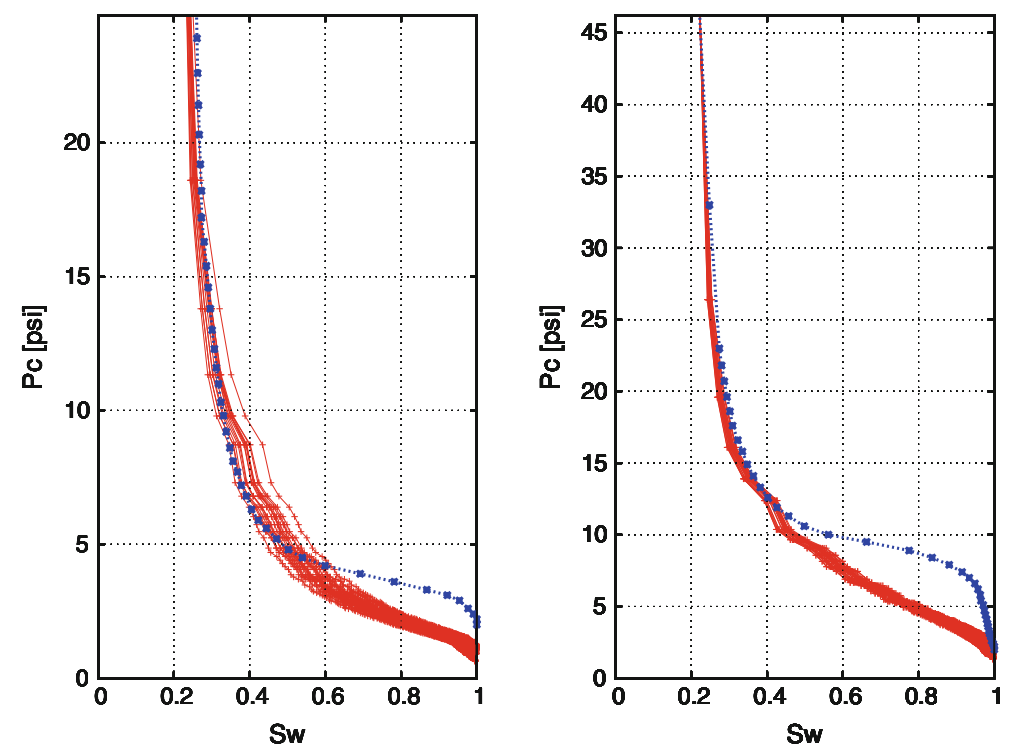

Fig. 15 The porosimetry capillary pressure curves for Frio (left) and Berea (right) sandstone samples. The plots include mercury drainage capillary pressure curves (dotted lines) for comparison

\subsection{MIS Porosimetry}

A mercury-injection capillary pressure curve, in combination with Eq. 2, is routinely used for evaluating the pore size distribution. This method involves drainage only, and practically eliminates the impact of capillary pressure hysteresis. However, the pore-size estimates can be affected by the contact angle uncertainty, associated with the roughness of the pore walls (Anderson 1987).

The MIS method offers an alternative approach to evaluating the pore-size distribution from the three-dimensional table of the maximal radii described in the previous section. This table is determined solely by the pore-space geometry as computed from the CT data. In particular, this table is not affected by the entry capillary pressure barrier. At the same time, the computational approach is limited by the resolution of the available CT image and the efficiency of the segmentation algorithm. For example, it does not account for the subresolution microporosity.

To illustrate the idea, we perform the computations on the same rock samples as in Figs. 13 and 14. To partially resolve the uncertainty associated with the subresolution microporosity, we rescale the saturation using the same value of $S_{0}$ which produced the data fit in the righthand-side plots in Figs. 13 and 14. Figure 15 shows the plots of the ordinary-percolation maximal-inscribed-spheres capillary pressure curves along with the curves computed from the mercury drainage data. Note that the computed curves do not have a plateaux like the ones based on the mercury injection data. Ordinary percolation curves may be associated with imbibition capillary pressure (Kumar et al. 2008). Figure 16 shows MIS-calculated cumulative pore size distributions for the same samples. The experimental curve almost overlays the computed one at saturations below $40 \%$. The point of junction apparently indicates the transition between the pore size distribution associated with the breakthrough penetration and the pore size distribution associated with mercury spreading after the breakthrough. 

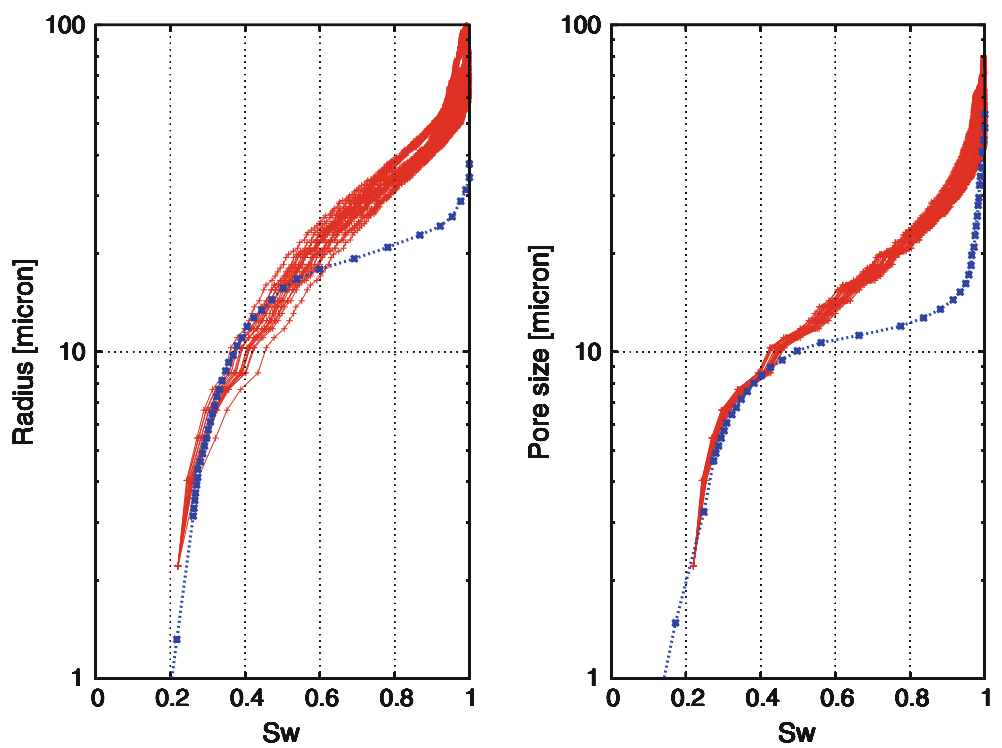

Fig. 16 Cumulative pore size distribution for Frio (left) and Berea sandstone samples (right). The dotted lines are the porosimetry curves based on mercury injection

\section{Summary and Conclusions}

The method of MIS models equilibrium of the two-phase fluid distributions in a porous medium. X-ray computer microtomography produces micron-scale 3D images of natural rock. These images are used as input data for MIS modeling and simulations. The direct analysis of the pore space geometry distinguishes this method from the pore network modeling approach, where the 3D image is used to represent the complex pore space geometry by a pore network. Simulations on a network of channels of simple geometries can be done in a very efficient way. However, the task of building a network representing the properties of a particular rock is far from trivial.

In this study, we have considered two applications of the MIS method: evaluation of fluid distributions in the pore space, and evaluation of the capillary pressure curves. The fluids are assumed to be in equilibrium dominated by the capillary forces. To exclude the difficulties of accounting for small dispersed bubbles, we assume that the nonwetting fluid phase satisfies the maximal occupancy requirement.

The simulations have been verified against experimental data. To verify the computation of equilibrium fluid distribution, we have used the data of the pioneering experiments that imaged the 3D fluid distribution in a Frio sandstone sample. These experiments have been performed by the second author at the ALS at Lawrence Berkeley National Laboratory. Even though the monochromatic electron beam offers great opportunities for microtomography of the pore space of natural rock, imaging of two fluid in the pore space of a rock sample constitutes a significant challenge. $\mathrm{A} \mathrm{CO}_{2}$ flood experiment on a small sample placed in a coreholder that fits into the imaging box is a great technical challenge. Reliable segmentation of the digital data requires a sufficiently high contrast between the phases. The densities of the fluids are much smaller than that of the solid, so the appropriate setting of the experiment to distinguish between the fluids is a delicate task. Even though the acquired images are fairly good for visual inspection, they include substantial noise, prohibiting computer-based pore 
space analysis. A number of customized cluster-search algorithms have been successfully applied to extract a rough characterization of the pore space geometry. The MIS simulations on that image reproduced well the local pore-scale fluid distribution. However, a big portion of the pore space was entirely bypassed by the injected $\mathrm{CO}_{2}$. We explain this observation by the local heterogeneities of the rock and the smallness of the domain available for simulations. Nevertheless, the successful verification of the pore-scale two-phase simulations confirms the approach used in the MIS simulations.

Verification of the MIS-derived capillary pressure curve has shown that this approach has predictive capabilities. However, the MIS modeling is limited by the image resolution and uncertainties in the segmentation of digital data. The computed drainage capillary pressure curves reproduce mercury injection data with a reasonable accuracy at high water saturations. The computations do capture the specific properties of different rocks, so that the computed capillary pressure curves for Frio and Berea sandstone samples are significantly different. Moreover, this difference mimics that in the mercury injection data.

Since the wetting fluid resides in small pores, pore corners and crevices, it has been assumed that the computed water distribution underestimates the actual water saturation. Addition of a constant saturation to account for the under-resolution effect results in a good match of the mercury injection capillary pressure curve. This obtained saturation has been applied to construct the MIS-based porosimetry curves. Although such curves suffer from the shortcomings of limited resolution, they help to resolve the ambiguity of mercury porosimetry curves, which mix information about the pore size distribution in the sample and the capillary barrier breakthrough information.

This study does not definitively answer the question what volume can be deemed representative for two-phase flow simulations. On the one hand, both pore-scale and reported here and core-scale $\mathrm{CO}_{2}$ flood experiments reported in Perrin and Benson (2010) show extremely non-uniform distribution of the $\mathrm{CO}_{2}$ saturation. On the other, the pore-scale simulations can predict capillary pressure curves that are in good agreement with the mercury porosimetry data. Apparently, the definition of a representative volume is task-dependent. We will return to this problem in the future.

The results presented here demonstrate potential of the pore-scale studies of multiphase fluid flow in natural rocks. X-ray microtomography produces digital data showing the distribution of the fluids in the pore space. Direct analysis of the pore space geometry by the MIS method could provide a modeling tool for interpretation of the pore-scale data with predictive capabilities.

Acknowledgements This work was partially supported by the U.S. Department of Energy's Assistant Secretary for Coal through the Zero Emission Research and Technology Program under US Department of Energy contract no. DE-AC02-05CH11231 to Lawrence Berkeley National Laboratory. Part of this work has been done while the first author was visiting the Energy Resources Engineering Department at Stanford University. The hospitality of this department and the Global Climate and Energy Project is gratefully appreciated. The first author also acknowledges partial support from the Research Partnership to Secure Energy for America. Portions of this work were performed at the ALS, Lawrence Berkeley National Laboratory, which is supported by the Office of Science, Office of Basic Energy Sciences, U.S. Department of Energy, under Contract No. DE-AC02-05CH11231. Special Core Analysis Laboratories, Inc. conducted the mercury injection experiments mentioned in this study.

Open Access This article is distributed under the terms of the Creative Commons Attribution Noncommercial License which permits any noncommercial use, distribution, and reproduction in any medium, provided the original author(s) and source are credited. 


\section{References}

Al-Futaisi, A., Patzek, T.W.: Impact of wettability on two-phase flow characteristics of sedimentary rock: Quasi-static model. Water Resour. Res. 39(2), 1042-1055 (2003)

Anderson, W.G.: Wettability literature survey. Part 4: effects of wettability on capillary pressure. J. Pet. Technol. 39(10), 1283-1300 (1987)

Apps, J.A.: A review of hazardous chemical species associated with $\mathrm{CO}_{2}$ capture from coal-fired power plants and their potential fate in $\mathrm{CO}_{2}$ geologic storage. Technical report, Lawrence Berkeley National Laboratory, Earth Sciences Division (2006)

Auzerais, F.M., Dunsmuir, J., Ferreol, B.B., Martys, N., Olson, J., Ramakrishnan, T.S., Rothman, D.H., Schwartz, L.M.: Transport in sandstone: a study based on three dimensional microtomography. Geophys. Res. Lett. 23, 705-708 (1996)

Bakke, S., Øren, P.E.: 3-D pore-scale modelling of sandstones and flow simulations in the pore networks. SPE J. 2, 136-149 (1997)

Bernard, D.: 3D quantification of pore scale geometrical changes using synchrotron computed microtomography. Oil Gas Sci. Technol. 60(5), 747-762 (2005)

Bico, J., Tordeux, C., Qur, D.: Rough wetting. Europhys. Lett. 55, 214-220 (2001)

Blunt, M.J.: Flow in porous media-pore-network models and multiphase flow. Curr. Opin. Colloid \& Interface Sci. 6(3), 197-207 (2001)

Blunt, M.J., King, P.: Relative permeabilities from two- and three-dimensional pore-scale metwork modeling. Transp. Porous Med. 6, 407-433 (1991)

Bryant, S., Blunt, M.: Prediction of relative permeability in simple porous-media. Phys. Rev. A 46, 20042011 (1992)

Bryant, S.L., King, P.R., Mellor, D.W.: Network model evaluation of permeability and spatial correlation in a real random sphere packing. Transp. Porous Med. 11, 53-70 (1993)

Chatzis, I., Dullien, F.A.L.: Mercury porosimetry curves of sandstones. Mechanisms of mercury penetration and withdrawal. Powder Technol. 29, 117-125 (1981)

Coles, M.E., Hazlett, R.D., Spanne, P., Muegge, E.L., Furr, M.J.: Characterization of reservoir core using computed microtomography. SPE J. 1(3), 295-302 (1996)

Coles, M.E., Hazlett, R.D., Muegge, E.L., Jones, K.W., Andrews, B., Dowd, Siddons, P., Peskin, A.: Developments in synchrotron X-ray microtomography with applications to flow in porous media. SPE Reserv. Eval. Eng. 1(4), 288-296 (1998a)

Coles, M.E., Hazlett, R.D., Spanne, P., Soll, W.E., Muegge, E.L., Jones, K.W.: Pore level imaging of fluid transport using synchrotron X-ray microtomography. J. Pet. Sci. Eng. 19, 55-63 (1998b)

Daley, T.M., Solbau, R.D., Ajo-Franklin, J.B., Benson, S.M.: Continuous active-source seismic monitoring of $\mathrm{CO}_{2}$ injection in a brine aquifer. Geophysics 72(5), A57-A61 (2007)

Derjagin, B.V., Churaev, N.V., Muller, V.M.: Surface forces. Plenum Press, New York (1987)

Dierick, M., Masschaele, B., Van Hoorebeke, L.: Octopus, a fast and user-friendly tomographic reconstruction package developed in LabView ${ }^{\circledR}$. Meas. Sci. Technol. 15, 1366-1370 (2004)

Doughty, C., Freifeld, B.M., Trautz, R.C.: Site characterization for $\mathrm{CO}_{2}$ geologic storage and vice versa: the Frio Brine Pilot, Texas, USA as a case study. Env. Geol. 54(8), 1635-1656 (2008)

Fatt, I.: The network model of porous media. 1. Capillary pressure characteristics. Trans. AIME 207(7), $144-159$ (1956a)

Fatt, I.: The network model of porous media. 2. Dynamic properties of a single size tube network. Trans. AIME 207(7), 160-163 (1956b)

Fatt, I.: The network model of porous media. 3. Dynamic propertries of networks with tube radius distribution. Trans. AIME 207(7), 164-181 (1956c)

Flukiger, F., Bernard, D.: A new numerical model for pore scale dissolution of calcite due to $\mathrm{CO}_{2}$ saturated water flow in 3D realistic geometry: principles and first results. Chem. Geol. 265(1-2), 171-180 (2009)

Huttenlocher, D.P., Klanderman, G.A., Rucklidge, W.J.: Comparing images using the Hausdorff distance. IEEE Trans. Pattern Anal. Mach. Intel. 15, 850-863 (1993)

Intergovernmental Panel on Climate Change (IPCC): Panel on climate change special report on carbon dioxide capture and storage. Cambridge University Press, Cambridge (2005)

Israelachvili, J.N.: Intermolecular and surface forces. 2nd edn. Academic Press, New York (1992)

Knackstedt, M.A., Sheppard, A.P., Sahimi, M.: Pore network modelling of two-phase flow in porous rock: the effect of correlated heterogeneity. Adv. Water Resour. 24, 257-277 (2001)

Kumar, M., Senden, T.J., Knackstedt, M.A., Latham, S., Pinczewski, W.V., Sok, R.M., Sheppard, A., Turner, M.L.: Imaging of core scale distribution of fluids and wettability. In: International Symposium of the Society of Core Analysts, Abu Dhabi, UAE (2008) 
Latham, S.J., Varslot, T.K., Sheppard, A.P.: Automated registration for augmenting micro-CT 3D images. In: Mercer G.N., Roberts A.J., (eds.) Proceedings of the 14th Biennial Computational Techniques and Applications Conference, CTAC-2008. ANZIAM J. 50, C534-C548 (2008)

Leverett, M.C.: Flow of oil-water mixtures through unconsolidated sands. Trans. AIME 132, 381-401 (1939)

Leverett, M.C.: Capillary behavior in porous solids. Trans. AIME 142, 152-169 (1941)

Leverett, M.C., Lewis, W.B., True, M.E.: Dimensional-model studies of oil-field behavior. Trans. AIME 146, 175-193 (1942)

Lindquist, W.B., Venkatarangan, A.: Investigating 3D geometry of porous media from high resolution images. Phys. Chem. Earth A 25(7), 593-599 (1999)

Luquot, L., Gouze, P.: X-ray microtomography characterization of hydrochemical properties changes induced by $\mathrm{CO}_{2}$ injection. Geochim. Cosmochim. Acta Suppl. 73, 804 (2009)

Muskat, M., Meres, M.W.: The Flow of Hetereogeneous Fluids through Porous Media 7, 346-363 (1936)

Noiriel, C., Gouze, P., Bernard, D.: Investigation of porosity and permeability effects from microstructure changes during limestone dissolution. Geophy. Res. Lett. 31, L24603 (2004)

Noiriel, C., Luquot, L., Mad, B., Raimbault, L., Gouze, P., van der Lee, J.: Changes in reactive surface area during limestone dissolution: an experimental and modelling study. Chem. Geol. 265(1-2), 160-170 (2009)

Øren, P.E., Bakke, S.: Reconstruction of Berea sandstone and pore-scale modelling of wettability effects. J. Pet. Sci. Eng. 39(3-4), 177-199 (2003)

Patzek, T.W.: Verification of a complete pore network simulator of drainage and imbibition. SPE J. 6(2), 144-156 (2001)

Patzek, T.W.: Subsurface sequestration of $\mathrm{CO}_{2}$ in the U.S: is it money best spent? Nat. Resour. Res. 19(1), 1-9 (2010)

Perrin, J.C., Benson, S.: An experimental study on the influence of sub-core scale heterogeneities on $\mathrm{CO}_{2}$ distribution in reservoir rocks. Transp. Porous Med. 82(1), 93-109 (2010)

Pomeau, Y., Villermaux, E.: Two hundred years of capillary research. Phys. Today 59(3), 39-44 (2006)

Prodanovic, M., Lindquist, W.B., Seright, R.S.: Porous structure and fluid partitioning in polyethylene cores from 3D X-ray microtomographic imaging. J. Colloid Interface Sci. 298, 282-297 (2006)

Prodanovic, M., Lindquist, W.B., Seright, R.S.: 3D image-based characterization of fluid displacement in a Berea core. Adv. Water Resour. 30, 214-226 (2007)

Purcell, W.R.: Capillary pressure-their measurements using mercury and the calculation of permeability therefrom. AIME Petroleum Transactions 185, 39-48 (1949)

Seright, R.S., Liang, J., Lindquist, W.B., Dunsmuir, J.H.: Characterizing disproportionate permeability reduction using synchrotron X-ray computed microtomography. SPE Form. Eval. Reserv. Eval. Eng. 5, 355364 (2002)

Sezgin, M., Sankur, B.: Survey over image thresholding techniques and quantitative performance evaluation. J. Electron Imag. 13, 146-165 (2004)

Silin, D.B.: On set-valued differentiation and integration. Set Valued Anal. 5(2), 107-146 (1997)

Silin, D.B., Patzek, T.W.: Pore space morphology analysis using maximal inscribed spheres. Phys. A Stat. Mech. Appl. 371, 336-360 (2006)

Spanne, P., Thovert, J.F., Jacquin, C.J., Lindquist, W.B., Jones, K.W., Adler, P.M.: Synchrotron computed microtomography of porous media: topology and transports. Phys. Rev. Lett. 73(14), 2001-2004 (1994)

Tomutsa, L., Silin, D., Radmilovic, V.: Analysis of chalk petrophysical properties by means of submicron-scale pore imaging and modeling. SPE Reserv. Eval. Eng. 10(3), 285-293 (2007)

Turner, M.L., Knufing, L., Arns, C.H., Sakellariou, A., Senden, T.J., Sheppard, A.P., Sok, R.M., Limaye, A., Pinczewski, W.V., Knackstedt, M.A.: Three-dimensional imaging of multiphase flow in porous media. Phys. A. Stat. Mech. Appl. 339, 166-172 (2004)

van Dijke, M.I.J., Piri, M., Helland, J.O., Sorbie, K.S., Blunt, M.J., Skjveland, S.M.: Criteria for three-fluid configurations including layers in a pore with nonuniform wettability. Water Resour. Res. 43, W12S05 (2007)

Vogel, H.J.: Digital unbiased estimation of the Euler-Poincaré characteristic in different dimensions. Acta Stereol. 16(2), 97-104 (1997)

Wyckoff, R.T., Botset, H.G.: The Flow of Gas-Liquid Mixtures through Unconsolidated Sands 7, 325-345 (1936)

Xu, B., Kamath, J., Yortsos, Y.C., Lee, S.H.: Use of pore-network models to simulate laboratory corefloods in a heterogeneous carbonate sample. SPE J. 4(4), 179-185 (1999)

Youssef, S., Bauer, D., Bekri, S., Rosenberg, E., Vizika, O.: Towards a better understanding of multiphase flow in porous media: 3D In-Situ fluid distribution imaging at the pore scale. In: International Symposium of the Society of Core Analysts, Noordwijk aan Zee, The Netherlands (2009) 